\title{
Permeation of Hydrophilic Molecules across Glycated Skin Is Differentially Regulated by the Stratum Corneum and Epidermis-Dermis
}

\author{
Mami Yokota and Yoshihiro Tokudome* \\ Laboratory of Dermatological Physiology, Faculty of Pharmaceutical Sciences, Josai University; 1-1 Keyakidai, \\ Sakado, Saitama 350-0295, Japan. \\ Received April 28, 2015; accepted June 14, 2015
}

\begin{abstract}
The effects of glycation on skin permeation and accumulation of compounds were evaluated using an in vitro glycated skin model. Glycation of the skin of hairless mice was induced using vertical diffusion cells and incubation with phosphate-buffered saline containing $50 \mathrm{~mm}$ glyoxal for $24 \mathrm{~h}$. Flux and accumulation in the skin were determined by applying hydrophilic and lipophilic molecules (Sodium fluorescein; FL-Na and Nile red, respectively) to this in vitro glycated skin model. Furthermore, to investigate the effect of glycation on epidermal-dermal barrier properties, we conducted diffusion experiments with FL-Na and fluorescein isothiocyanate-dextran using stratum corneum (SC)-stripped glycated skin. The in vitro glycated skin model demonstrated characteristic glycation alterations like a yellowish change in skin color and surface roughness. For low-molecular weight (MW) hydrophilic molecules, flux across glycated full-thickness skin was higher than that across normal skin, although there was no difference with lipophilic molecules. However, glycated epidermis-dermis showed lower flux, and the difference increased with the MW of the compound. Furthermore, the amount of high-MW hydrophilic molecules accumulated in glycated epidermis-dermis was decreased. These results suggest that glycated SC and epidermis-dermis differentially regulate the permeability of hydrophilic molecules and highlight the importance of controlling drug delivery by modifying the formulation or method of application depending on skin condition.
\end{abstract}

Key words skin permeation; advanced glycation end product; stratum corneum

As a route of drug administration, the skin attracts much attention due to non-invasive application, ease of dose control and absorption without hepatic first-pass metabolism. However, human skin changes over time due to chronological aging, exposure to UV radiation, and diseases such as atopic dermatitis and psoriasis. With the increase in the aging population in recent decades, there is a growing need to care for a variety of skin types. Therapeutic applications involving the skin have also increased in diversity in recent years. A variety of chemical and physiological methods are being studied to lower the barrier posed by the skin's outermost layer, the stratum corneum (SC). ${ }^{1}$ Consisting of corneocytes and intercellular lipids, the SC plays an important role as a rate limiting layer for the penetration of compounds. Serving as the major protection from foreign chemicals and water loss, and also associated with body temperature regulation, the SC is permeable to only lipophilic and $<500 \mathrm{Da}$ compounds. ${ }^{2}$ ) Therefore, various biochemical approaches to increase permeability across the SC have been attempted over the years. ${ }^{3)}$ Physical methods such as microneedles, thermal ablation and microdermabrasion are also used to enhance the efficiency of penetration by piercing or removing the SC. Additionally, iontophoresis and sonophoresis increase skin permeability by electrical driving force and ultrasound, respectively. ${ }^{4}$

Despite many percutaneously applied cosmetics and drugs being used to treat damaged skin or diseases that affect skin condition, few reports have shown a difference in penetration and permeation between normal and damaged skin. ${ }^{5-7)}$

Advanced glycation end products (AGEs), which are linked to both aging and hyperglycemia, cause marked functional and structural alterations in human skin. ${ }^{8,9}$ AGEs are gener- ated via the non-enzymatic Maillard reaction between reducing sugars and proteins, lipids or nucleic acids. ${ }^{10,11)}$ Because glycated proteins tend to be resistant to proteinases, they accumulate in tissues with glycation stress, including due to aging and diabetes mellitus (DM). Skin accumulation of AGEs has been detected in both the epidermis and dermis. ${ }^{9,12}$ ) In the epidermis, glycated keratin leads to a yellowish change in skin color and decrease in water content of the SC, resulting in skin dryness. ${ }^{13)}$ Moreover, glycation of dermal collagen and elastic fibers contributes to stiffness and loss of elasticity, forming wrinkles. ${ }^{14,15)}$ In addition to the modification of structural proteins, AGEs also induce biological reactions via binding to receptor for AGEs (RAGE) ${ }^{16)}$ Binding of AGEs to RAGE induces activation of nuclear factor kappa B (NF$\kappa \mathrm{B})$ and subsequent transcription of proinflammatory genes including interleukin 1 (IL-1), tumor necrosis factor alpha (TNF $\alpha$ ), matrix metalloproteinases (MMPs) and RAGE itself, accelerating further skin aging and deconstruction. ${ }^{17,18)}$ For these reasons, it is expected that alteration of the membrane properties of glycated skin causes changes in its permeability to compounds.

To clarify the effect of skin glycation on the permeation of compounds, we established a simple in vitro model of glycated skin. This model has the features of aged skin including a yellowish and rough macroscopic appearance. Using this model, we investigated permeation of sodium fluorescein (FL-Na) and fluorescein isothiocyanate-dextran (FD4) as hydrophilic model molecules, and Nile red as a lipophilic model molecules. Furthermore, we investigated the contribution of the $\mathrm{SC}$ to differences in permeation between normal and glycated skin. 


\section{MATERIALS AND METHODS}

Reagents Glyoxal (GO), guanidine hydrochloride, sodium fluorescein and Nile red were purchased from Wako Pure Chemical Industries, Ltd. (Osaka, Japan). Fluorescein isothiocyanate-dextran was purchased from Sigma (St. Louis, MO, U.S.A.). Primary anti-AGEs antibody (clone 6D12) was purchased from TransGenic Inc. (Kumamoto, Japan). Alexa 546-conjugated secondary antibody was purchased from Life Technologies (Carlsbad, CA, U.S.A.).

Animals Seven- to nine-week-old male hairless mice (Hos: HR-1) were purchased from Hoshino Experiment Animal Center (Bando, Ibaraki, Japan). All animal experiments were carried out in accordance with the guidelines of, and approved by, the Animal Research Committee of Josai University.

Induction of Glycation and Diffusion Experiments Skin samples removed from mice and cleaned of subcutaneous tissue and fat were mounted on vertical diffusion cells. The cells used in this study had an effective diffusion area of $1.77 \mathrm{~cm}^{2}$ and a receptor compartment volume of $5.0 \mathrm{~mL}$. For induction of glycation, the skin specimens were hydrated with phosphate-buffered saline (PBS) (control), PBS containing GO $10 \mathrm{~mm}, 50 \mathrm{~mm}$ (AGEd skin model) or $50 \mathrm{~mm}$ GO with $1 \%$ guanidine hydrochloride for $24 \mathrm{~h}$. Glycation was induced from dermal side. The receptor fluid was maintained at $32^{\circ} \mathrm{C}$, and continuously agitated with a magnetic stirrer bar. After glycation induction, autofluorescence of receptor fluids, which is indicator of formation of AGEs, were measured at an excitation wavelength of $365 \mathrm{~nm}$ and an emission wavelength of $450 \mathrm{~nm}$.

For diffusion experiment, receptor fluid was removed and replaced with the appropriate fluids. For diffusion experiments across full-thickness skin, $1 \mathrm{~mm}$ of FL-Na or $0.3 \mathrm{~mm}$ of Nile red in $50 \%$ methanol was used as the donor fluid and $50 \%$ methanol was used as the receptor fluid. For diffusion experiments across SC-stripped skin, $1 \mathrm{~mm}$ of FL-Na and $0.25 \mathrm{~mm}$ of FD4 in PBS were used as donor fluid and PBS was used as the receptor fluid. At time zero, $1.5 \mathrm{~mL}$ of donor fluid was applied to the upper surface of the skin in the donor chamber of the vertical diffusion cell. At appropriate intervals, $300-\mu \mathrm{L}$ aliquots were removed from the receptor fluid and replaced with an equal volume of fluid. To determine the quantity of FL-Na and FD4, the fluorescence was measured at an excitation wavelength of $490 \mathrm{~nm}$ and emission wavelength of $520 \mathrm{~nm}$ using a SpectraMax M2 $2^{e}$ (Molecular Devices, Sunnyvale, CA, U.S.A.). Nile red was measured at an excitation wavelength of $570 \mathrm{~nm}$ and an emission wavelength of $650 \mathrm{~nm}$.

Measurement of AGEs Indicator and Compounds in Skin For measurement of autofluorescence derived from AGEs, skin samples were collected immediately after finishing glycation induction. Washed with purified water, skin samples were minced in 50\% methanol and sonicated for $20 \mathrm{~min}$. Following centrifugation, the supernatant was used for measurement of the autofluorescence of the skin at a wavelength as per previous section. For measurement of compounds in skin, skin samples were collected $6 \mathrm{~h}$ after application of each compounds as written above. Accumulation of compounds in the skin were measured at the appropriate wavelength, as per previous section.

\section{Macroscopic Observations}

Colorimetry

Skin color was measured using a chromameter CR-400 (Konica-Minolta, Tokyo, Japan). The measurement of skin samples was performed by placing the skin samples on a white board. Data are expressed in the $L^{*} a^{*} b^{*}$ color space, and the parameter $b^{*}$ was employed as the value for yellow color.

\section{Profilometry}

The three-dimensional optical measurement of each skin section was performed using PRIMOS (GF Messtechnik $\mathrm{GmbH}$, Berlin, Germany). Three parameters, Ra (roughness average), Rz (10-point height) and Ry (maximum height of the profile), were used as indicators of skin roughness.

Immunohistochemistry Skin specimens were embedded in OCT compound (Sakura Finetek Japan Co., Ltd., Tokyo, Japan), snap-frozen in liquid nitrogen, and stored at $-80^{\circ} \mathrm{C}$. Frozen samples were cut into $8-\mu \mathrm{m}$-thick sections, and used for immunohistochemical analysis. The sections were washed with running water for $5 \mathrm{~min}$ then fixed with ice cold methanol, for $15 \mathrm{~min}$ at $-30^{\circ} \mathrm{C}$. Nonspecific staining was blocked by pre-incubation with $1 \%$ bovine serum albumin (BSA) in PBS for $1 \mathrm{~h}$ at room temperature. For primary antibody reaction, skin sections were incubated with the antibody against AGEs at a 1:200 dilution in Can Get Signal immunostain ${ }^{\circledR}$ Solution A (TOYOBO, Osaka, Japan) overnight at $4^{\circ} \mathrm{C}$. Bound antibodies were visualized with Alexa 546-conjugated secondary antibody at a 1:200 dilution in Can Get Signal immunostain $^{\circledR}$ solution $A$ for $1 \mathrm{~h}$ at room temperature. After washing, hoechst33258 (Dojindo Laboratories, Mashiki, Kumamoto, Japan) was added for nuclear counterstaining. All images were obtained using an IX71 microscope (Olympus, Tokyo, Japan).

Preparation of Stratum Corneum Samples Samples were collected of the 1st layer of the SC from glycated skin by tape stripping using cellophane tape (Nichiban, Tokyo, Japan). SC samples were fixed onto glass slides. After taping SC to the slides, the cellophane tape was dissolved in xylene overnight. After washing with ethanol and drying, the SC samples were permeabilized with $0.05 \%$ Tween 20 in PBS for $30 \mathrm{~min}$ at room temperature and blocked with $1 \%$ BSA in PBS. Slides were subsequently processed for immunohistochemistry (as per previous section)

Data Analysis Statistical analysis was performed using SAS statistics software version 9.2 (SAS Institute, Cary, NC, U.S.A.). Differences between groups were assessed by means of paired Student's $t$-test or Tukey's test and a $p$ value of $<0.05$ was considered as the limit of statistical significance.

\section{RESULTS}

Characterization of in Vitro Glycated Skin To elucidate the difference in cutaneous permeation between normal and glycated skin, we established a simple method to induce skin glycation using a vertical diffusion cell. In this in vitro model, using receptor fluid which contains the glycation inducer $\mathrm{GO}$, glycation was induced from the basal side of the skin. As shown in Fig. 1a, after 24h, accumulation of AGEs was observed in all layers of the skin, including the SC. In agreement with these results, fluorescent intensity of receptor fluids and skin at an excitation wavelength of $365 \mathrm{~nm}$ and emission wavelength of $450 \mathrm{~nm}$, reported as an indicator of AGEs for- 
a
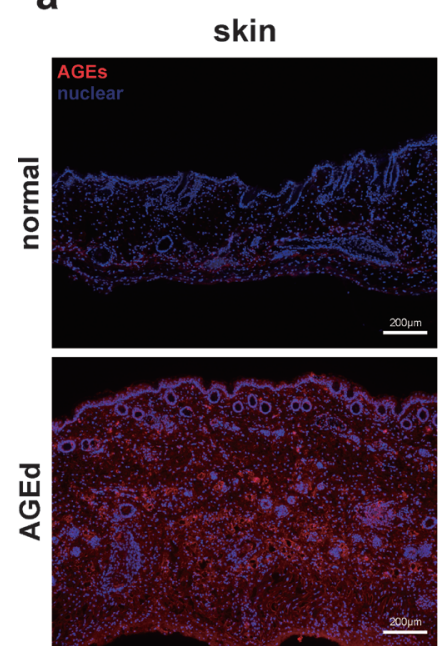
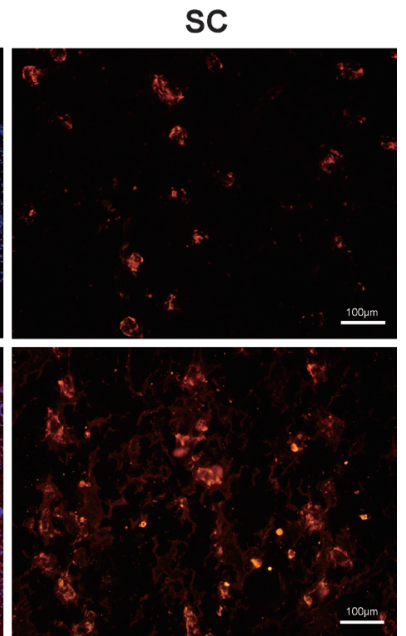

b

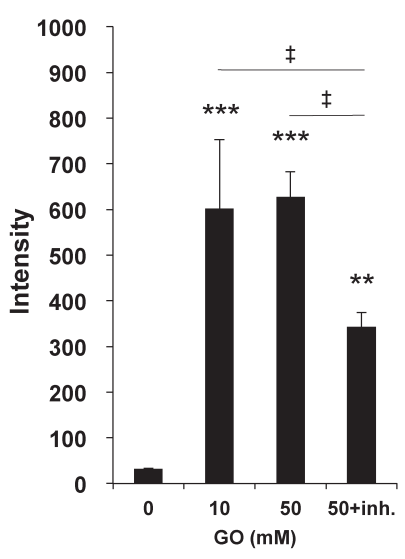

C

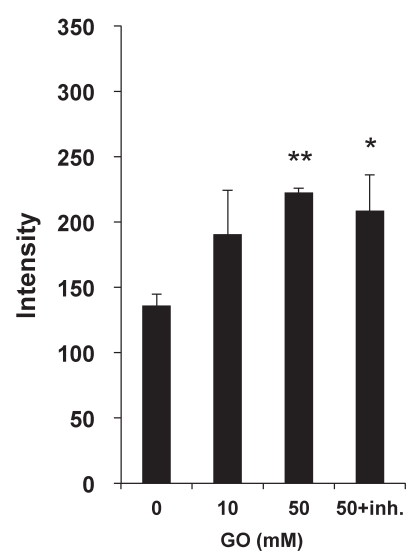

Fig. 1. Confirmation of Glycation in an in Vitro Glycated Skin Model

Immunohistochemistry of skin and SC with anti-AGEs antibody. Glycation was induced by hydration with $0 \mathrm{~mm}$ GO (normal) or $10 \mathrm{~mm}$ (for skin), $50 \mathrm{~mm}$ (for SC) GO (AGEd) in PBS at $32^{\circ} \mathrm{C}$ for $24 \mathrm{~h}$ (a). Intensity of fluorescence (excitation wavelength $365 \mathrm{~nm}$, emission wavelength $450 \mathrm{~nm}$ ) in receptor fluids (b) and skin homogenates (c). Glycation was induced by hydration with the indicated concentration of GO and inhibitor (inh.) in PBS at $32^{\circ} \mathrm{C}$ for $24 \mathrm{~h}$. All results are expressed as the mean \pm S.D. of $n=3$ replicates. ${ }^{*} p<0.05,{ }^{*} p<0.05, * * p<0.01, * * * p<0.001$.

a

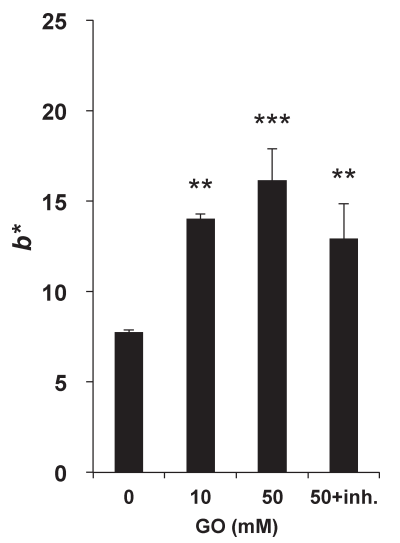

b

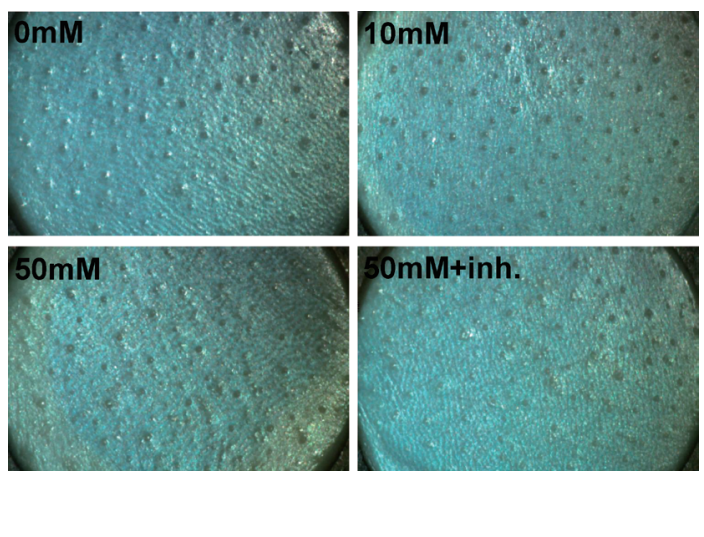

Fig. 2. Macroscopic Properties of in Vitro Glycated Skin Model

Yellowish color change was evaluated by colorimetry and expressed in $b^{*}$. Glycation was induced by hydration with indicated concentration of GO and inhibitor (inh.) in PBS at $32^{\circ} \mathrm{C}$ for $24 \mathrm{~h}$. (a). Surface appearance of glycated skin after $24 \mathrm{~h}$ incubation under the indicated conditions (b). All results are expressed as mean \pm S.D. of $n=3$ replicates. ${ }^{* *} p<0.01, * * * p<0.001$.

mation, ${ }^{19)}$ increased in a GO concentration dependent manner. With the addition of guanidine hydrochloride, a known inhibitor of glycation, the fluorescence intensity of the receptor fluid was significantly decreased, but not that of skin homogenates (Figs. 1b, c).

In parallel with the glycation confirmation study, further analysis focusing on the phenotype of glycated skin was performed to clarify how glycation changes the features of the skin in this model. One of the most distinguishable characteristics of glycated skin is its color change to yellow. ${ }^{20)}$ As shown in Figs. 2a and b, glycated skin showed a yellowish tinge. The evaluation of skin profilometry revealed that in vitro glycation also induced skin surface roughness, but there was no significant difference between each group (Fig. 2b, Table 1).

Cutaneous Permeation of Low Molecular Weight Compounds across Full-Thickness Glycated Skin After confirming glycation, we determined the flux of compounds
Table 1. Surface Profilometry of the in Vitro Glycated Skin Model

\begin{tabular}{lrccr}
\hline \hline & $0 \mathrm{~mm}$ & $10 \mathrm{~mm}$ & $50 \mathrm{~mm}$ & $50 \mathrm{~mm}+\mathrm{inh}$. \\
\hline $\mathrm{Ra}$ & $8.2 \pm 0.4$ & $9.7 \pm 1.5$ & $9.6 \pm 0.1$ & $9.0 \pm 0.2$ \\
$\mathrm{Rz}$ & $42.5 \pm 2.1$ & $47.5 \pm 8.1$ & $47.6 \pm 0.5$ & $44.8 \pm 1.7$ \\
$\mathrm{Ry}$ & $61.4 \pm 8.8$ & $67.0 \pm 17.8$ & $63.6 \pm 3$ & $62.6 \pm 6.3$ \\
\hline
\end{tabular}

across skin. FL-Na (MW 376.28) and Nile red (MW 318.37) were used as hydrophilic and lipophilic molecules, respectively. Skin glycation induced significantly higher permeation of FL-Na compared with normal skin after application for $6 \mathrm{~h}$ (Fig. 3a), with a flux ratio AGEd/normal $_{\text {of }} 2.09$ (Table 2). However, accumulation of FL-Na in glycated skin $6 \mathrm{~h}$ after application showed no significant difference compared with normal skin (Table 2). In contrast, Nile red showed no difference in permeation across and accumulation in skin between normal and glycated one (Fig. 3b, Table 2). 
a

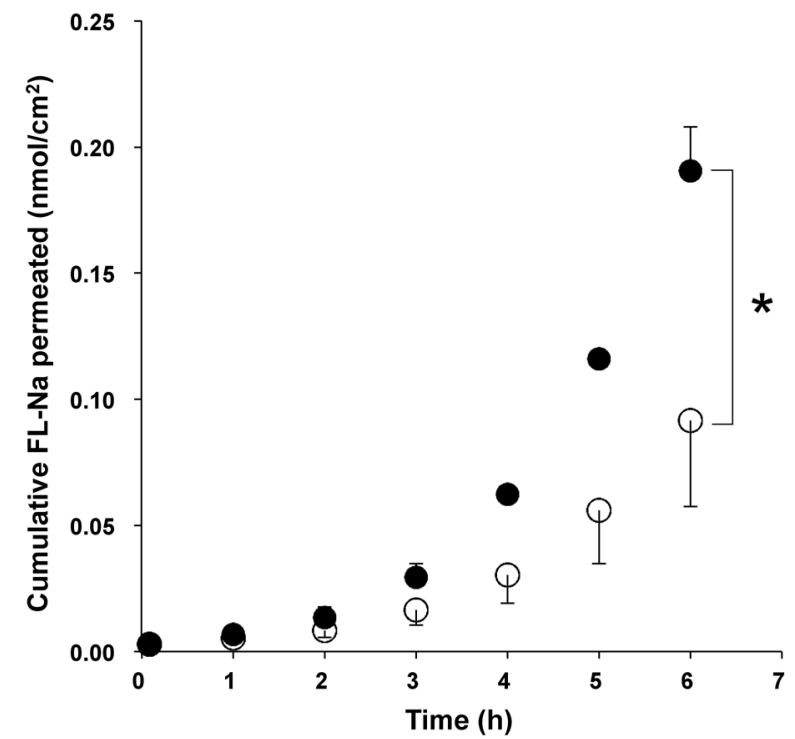

b

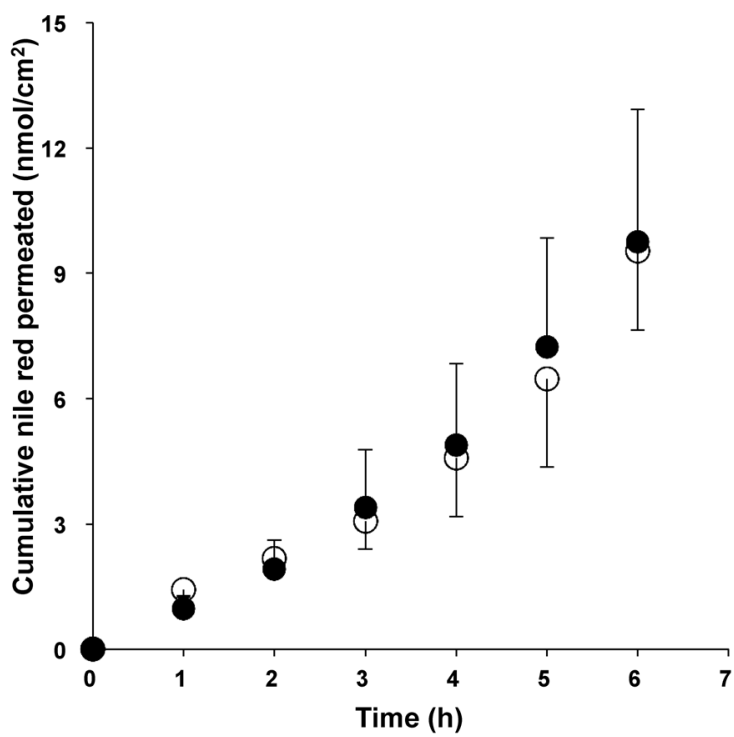

Fig. 3. Cutaneous Permeation of Low Molecular Weight Molecules across Full-Thickness Glycated Skin

Cumulative amount of FL-Na (a) and Nile red (b) permeating across full-thickness normal (O) and AGEd (O) skin. $1 \mathrm{~mm}$ of FL-Na or $0.3 \mathrm{~mm}$ of Nile red in $50 \%$ methanol was used as the donor solution. $50 \%$ methanol was used as the receptor solution. All results are expressed as mean \pm S.D. of $n=3$ replicates. $* p<0.05$.

Table 2. Parameters of Skin Permeation across the in Vitro Glycated Skin Model

\begin{tabular}{cccccc}
\hline \hline Skin & Compounds & Condition & Flux $\left(\mathrm{nmol} / \mathrm{cm}^{2} / \mathrm{h}\right)$ & Flux ratio (AGEd/normal) & Contents in skin $\left(\mathrm{nmol} / \mathrm{cm}^{2}\right)$ \\
\hline \multirow{2}{*}{ Full-thickness } & FL-Na & Normal & $0.03 \pm 0.01$ & - & $2.70 \pm 0.54$ \\
& & AGEd & $0.06 \pm 0.01^{\#}$ & 2.09 & $2.22 \pm 0.22$ \\
& Nile red & Normal & $2.48 \pm 0.41$ & - & $4.99 \pm 1.27$ \\
& & AGEd & $2.43 \pm 0.61$ & 0.98 & $4.37 \pm 0.52$ \\
Epidermis-dermis & FL-Na & Normal & $98.00 \pm 7.59$ & - & $5.99 \pm 1.34$ \\
& & AGEd & $62.44 \pm 14.48^{*}$ & 0.64 & $4.64 \pm 1.11$ \\
& FD4 & normal & $1.76 \pm 0.27$ & 0.40 & $6.03 \pm 0.95$ \\
& & AGEd & $0.71 \pm 0.09^{* *}$ & $2.60 \pm 0.85^{*}$ \\
\hline
\end{tabular}

Flux ratio ${ }_{\mathrm{AGEd} / \text { normal }}$ means: Flux across glycated skin / Flux across normal skin. Student's $t$-test was performed between the results from normal and AGEd skin. ${ }^{\#} p=0.05$, $* p<0.05, * * p<0.01$.

Cutaneous Permeation of Hydrophilic Compounds across Stratum Corneum-Stripped Glycated Skin We next sought to determine what causes the difference in permeation between normal and glycated skin. Because SC was the penetration regulating layer, we performed diffusion experiment using tape-stripped skin. First, we confirmed that 15 times tape stripping completely removed the SC (data not shown). Flux of FL-Na across epidermis-dermis was approximately 3000 times higher than full-thickness skin (Fig. 4a, Table 2). In contrast to full-thickness permeation, permeation across epidermis-dermis was significantly lower in glycated skin after application for $6 \mathrm{~h}$, with a flux ratio ${ }_{\mathrm{AGEd} / \text { normal }}$ of 0.64 (Fig. 4a, Table 2). The amount of FL-Na in glycated epidermis-dermis showed a tendency to be lower than normal epidermis-dermis, but not significantly so (Table 2). To examine the barrier properties of epidermis-dermis in detail, we assessed skin flux of FD4 (average MW 4000). Consistent with the result of FL-Na, glycated epidermis-dermis showed lower flux to FD4. However, FD4 had a lower flux ratio AGEd/normal (0.40) compared with that of FL-Na (Fig. 4b, Table 2), and the amount of FD4 in the skin was also significantly lower in glycated skin (Table 2).

\section{DISCUSSION}

In the field of percutaneous drug application, it is not necessarily the case that drugs and cosmetics are only applied to healthy skin. In spite of this fact, there are only a limited number of reports on permeation of compounds across damaged or diseased skin. In the present study, we established a simple in vitro model of glycated skin, which is relevant to both DM and aging, and clarified the permeation profile of model molecules.

In vitro skin glycation was induced by GO in vertical diffusion cells. GO, reactive dicarbonyl compounds produced by normal cell metabolism, lead to protein adducts and crosslinks. ${ }^{21)}$ The plasma concentration of GO in human is 0.1 to $1 \mu \mathrm{M}$ and that patients with diabetes or kidney failure show higher levels, inducing glycation over a long period of time. ${ }^{22)}$ In this model, though glycation was induced at an accelerated rate with high concentrations of GO from the dermal side, AGEs were detected in the full-thickness of the skin, includ- 
a

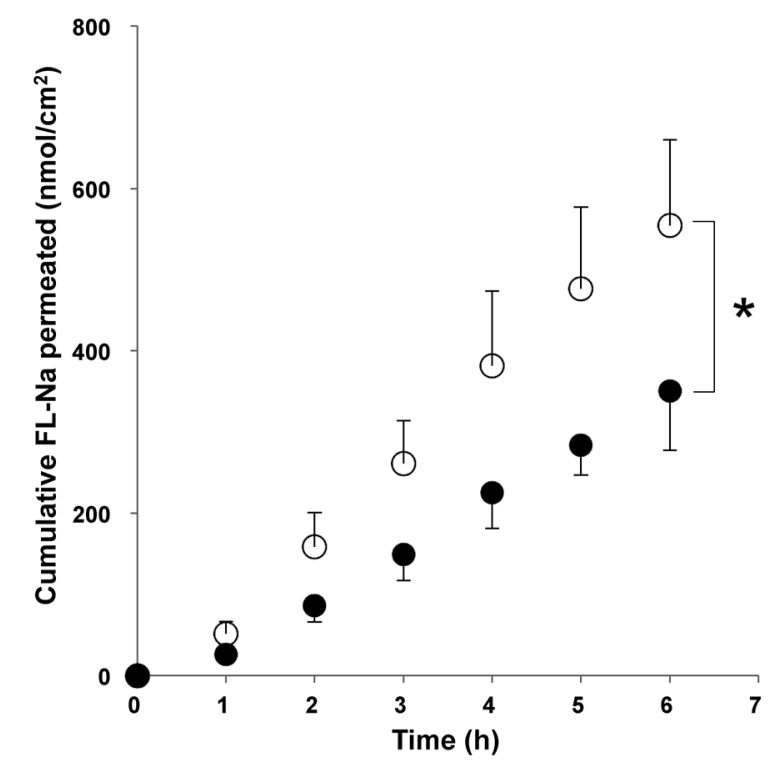

b

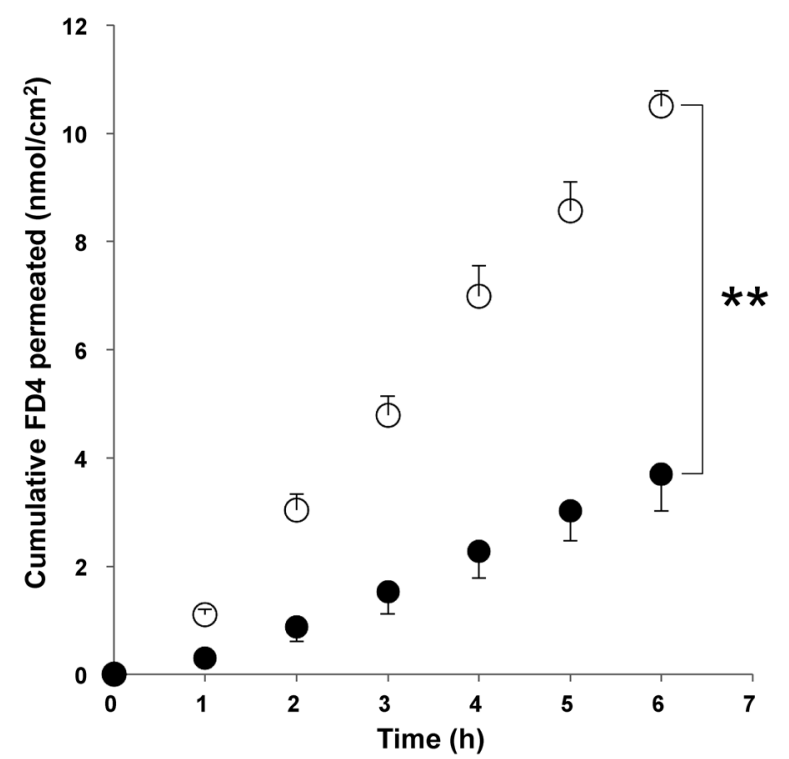

Fig. 4. Cutaneous Permeation of Hydrophilic Molecules across Glycated Epidermis-Dermis

Cumulative amount of FL-Na (a) and FD4 (b) permeating across normal (O) and AGEd ( $)$ epidermis-dermis. 1 mm of FL-Na and 0.25 mM of FD4 in PBS were used as donor solutions. PBS was used as the receptor solution. All results are expressed as mean \pm S.D. of $n=3$ replicates. ${ }^{*} p<0.05, * * p<0.01$.

ing SC, after $24 \mathrm{~h}$ (Fig. 1a). It is well known that increases in the $b^{*}$ value and autofluorescence reflect the accumulation of AGEs in skin. ${ }^{19,20)}$ Glycation induced by $50 \mathrm{~mm}$ GO showed 1.6 times higher autofluorescence and 2.1 times higher $b^{*}$ values than normal skin (Figs. 2a, b). Because these results were close to clinical experimental values, ${ }^{23}$ this experimental setup was considered reasonable for evaluation of skin permeability of compounds. Furthermore, previous studies showed that wrinkles in glycated skin were formed by destruction of extracellular matrix via activation of MMPs secreted by keratinocytes and fibroblasts. ${ }^{24)}$ However, in this model, structural change by modification may be responsible for surface roughness (Table 1).

To examine the permeation profile of hydrophilic and lipophilic compounds across glycated skin, we performed diffusion experiments. The cumulative amount of FL-Na was higher across glycated skin, whereas Nile red permeation showed no difference (Figs. 3a, b, Table 2). It is well documented that percutaneous penetration occurs thorough intracellular, transcellular, and to a lesser extent, appendageal routes. ${ }^{25)}$ Notably, hydrophilic molecules diffuse across the polar head of the lipid as a major route. As only hydrophilic compounds showed higher permeation in glycated skin, it is thought that modification of the polar head of intercellular lipids by AGEs derived from GO causes barrier disruption, leading to an increase in permeation rate.

Viable epidermis and dermis are regarded as important barriers to cutaneous absorption. ${ }^{26,27)}$ The flux ratio $_{\mathrm{FD} 4 / \mathrm{FLNa}}$ across normal epidermis-dermis was 0.07 (Table 2). This result indicates the existence of epidermal-dermal barrier function depending on the MW against cutaneously applied compounds. In accordance with these reports, our results suggest the importance of epidermal-dermal barrier after the removal of SC. Additionally, this study investigated the effect of glycation on epidermal-dermal permeation and found that glycated epidermis-dermis could be a tighter barrier to hydrophilic molecules rather than normal one (Figs. 4a, b, Table 2). One of the reasons why full-thickness skin and SC stripped skin showed opposite permeation profile is inferred change in hydrophilic property of dermal layer. GO prefer to react with lysine and arginine, which are basic hydrophilic amino acid, forming carbonylated modification. This reaction could make dermis more hydrophobic environment, causing decrease in permeation across and contents of compounds in glycated skin (Table 2). Together with the results from full-thickness skin, it is suggested that (1) glycated SC regulates the permeation of compounds and (2) glycated SC promotes, while epidermisdermis inhibits, permeation of hydrophilic molecules.

Though further studies are needed to explore how glycation causes these phenomena, it would be important to control permeability of compounds across glycated skin. Not only percutaneously applied cosmetics and drugs, percutaneous absorption of pharmaceutical preparations with hydrophilic high molecular weight active compounds are currently under development, based on the technology of iontophoresis, sonophoresis or microneedles. In the near future, promising non-invasive administration of these compounds could be made more effective by considering formulations depending on glycation level of the skin, as predicted by $b^{*}$ value and autofluorescence.

At the actual cellular level, AGEs induce chronic inflammation leading to disruption of the well-organized skin differentiation process, as well as structural changes. However, this study could not reflect such physiological changes. To provide effective transdermal drug delivery system to diverse types of diseased or damaged skin, including glycated skin, further interdisciplinary research is essential.

\section{CONCLUSION}

The in vitro glycated skin model prepared from hairless 
mice could be used to predict permeability and accumulation of compounds in glycated skin. Notably, low molecular weight, hydrophilic molecules have different permeation profiles between normal and glycated skin. Although the stratum corneum serves as a major rate limiting layer for permeability of compounds in glycated skin, the epidermis-dermis can also be a factor regulating the behavior of compounds applied to skin. This study highlights the importance of considering the difference in permeability according to the method of application and target site when thinking about percutaneous application via glycated skin.

Conflict of Interest The authors declare no conflict of interest.

\section{REFERENCES}

1) Prausnitz MR, Langer R. Transdermal drug delivery. Nat. Biotechnol., 26, 1261-1268 (2008).

2) Bos JD, Meinardi MM. The 500 Dalton rule for the skin penetration of chemical compounds and drugs. Exp. Dermatol., 9, 165-169 (2000).

3) Murthy SN. Transdermal drug delivery: approaches and significance. $R R T D, 2012,1-2$ (2012).

4) Schoellhammer CM, Blankschtein D, Langer R. Skin permeabilization for transdermal drug delivery: recent advances and future prospects. Expert Opin. Drug Deliv., 11, 393-407 (2014).

5) Chiang A, Tudela E, Maibach HI. Percutaneous absorption in diseased skin: an overview. J. Appl. Toxicol., 32, 537-563 (2012).

6) Hung CF, Fang CL, Al-Suwayeh SA, Yang SY, Fang JY. Evaluation of drug and sunscreen permeation via skin irradiated with UVA and UVB: comparisons of normal skin and chronologically aged skin. $J$. Dermatol. Sci., 68, 135-148 (2012).

7) Jakasa I, Verberk MM, Esposito M, Bos JD, Kezic S. Altered penetration of polyethylene glycols into uninvolved skin of atopic dermatitis patients. J. Invest. Dermatol., 127, 129-134 (2007).

8) Crisan M, Taulescu M, Crisan D, Cosgarea R, Parvu A, Cãtoi C, Drugan T. Expression of advanced glycation end-products on sunexposed and non-exposed cutaneous sites during the ageing process in humans. PLoS ONE, 8, e75003 (2013).

9) Schleicher ED, Wagner E, Nerlich AG. Increased accumulation of the glycoxidation product $N^{E}$-(carboxymethyl)lysine in human tissues in diabetes and aging. J. Clin. Invest., 99, 457-468 (1997).

10) Gkogkolou P, Bohm M. Advanced glycation end products: Key players in skin aging? Dermatoendocrinology, 4, 259-270 (2012).

11) Ichihashi $M$, Yagi $M$, Nomoto $K$, Yonei $Y$. Glycation stress and photo-aging in skin. ANTI-AGING MEDICINE, 8, 23-29 (2011).

12) Kawabata $K$, Yoshikawa $H$, Saruwatari K, Akazawa $Y$, Inoue T, Kuze T, Sayo T, Uchida N, Sugiyama Y. The presence of $N^{z}-$ (carboxymethyl)lysine in the human epidermis. Biochim. Biophys. Acta, 1814, 1246-1252 (2011).

13) Sakai S, Kikuchi K, Satoh J, Tagami H, Inoue S. Functional properties of the stratum corneum in patients with diabetes mellitus: similarities to senile xerosis. Br. J. Dermatol., 153, 319-323 (2005).

14) Verzijl N, DeGroot J, Thorpe SR, Bank RA, Shaw JN, Lyons TJ, Bijlsma JW, Lafeber FP, Baynes JW, TeKoppele JM. Effect of collagen turnover on the accumulation of advanced glycation end products. J. Biol. Chem., 275, 39027-39031 (2000).

15) Yoshinaga E, Kawada A, Ono K, Fujimoto E, Wachi H, Harumiya S, Nagai R, Tajima S. $N^{\varepsilon}$-(Carboxymethyl)lysine modification of elastin alters its biological properties: implications for the accumulation of abnormal elastic fibers in actinic elastosis. J. Invest. Dermatol., 132, 315-323 (2012).

16) Lohwasser C, Neureiter D, Weigle B, Kirchner T, Schuppan D. The receptor for advanced glycation end products is highly expressed in the skin and upregulated by advanced glycation end products and tumor necrosis factor-alpha. J. Invest. Dermatol., 126, 291-299 (2006).

17) Leibold JS, Riehl A, Hettinger J, Durben M, Hess J, Angel P. Keratinocyte-specific deletion of the receptor RAGE modulates the kinetics of skin inflammation in vivo. J. Invest. Dermatol., 133, 2400-2406 (2013).

18) Zhu P, Ren M, Yang C, Hu YX, Ran JM, Yan L. Involvement of RAGE, MAPK and NF-kappaB pathways in AGEs-induced MMP-9 activation in HaCaT keratinocytes. Exp. Dermatol., 21, 123-129 (2012).

19) Monnier VM, Vishwanath V, Frank KE, Elmets CA, Dauchot P, Kohn RR. Relation between complications of type I diabetes mellitus and collagen-linked fluorescence. N. Engl. J. Med., 314, 403-408 (1986).

20) Ogura Y, Kuwahara T, Akiyama M, Tajima S, Hattori K, Okamoto K, Okawa S, Yamada Y, Tagami H, Takahashi M, Hirao T. Dermal carbonyl modification is related to the yellowish color change of photo-aged Japanese facial skin. J. Dermatol. Sci., 64, 45-52 (2011).

21) Sady C, Jiang CL, Chellan P, Madhun Z, Duve Y, Glomb MA, Nagaraj RH. Maillard reactions by $\alpha$-oxoaldehydes: detection of glyoxal-modified proteins. Biochim. Biophys. Acta, 1481, 255-264 (2000).

22) Kielhorn J, Pohlenz-Michel C, Schmidt S, Mangelsdorf I. Concise International Chemical Assessment Document 57. World Health Organization, Geneva, 2004.

23) Mulder DJ, Water TV, Lutgers HL, Graaff R, Gans RO, Zijlstra F, Smit AJ. Skin autofluorescence, a novel marker for glycemic and oxidative stress-derived advanced glycation endproducts: an overview of current clinical studies, evidence, and limitations. Diabetes Technol. Ther., 8, 523-535 (2006).

24) Pageon H, Bakala H, Monnier VM, Asselineau D. Collagen glycation triggers the formation of aged skin in vitro. Eur. J. Dermatol., 17, 12-20 (2007).

25) Barry BW. Mode of action of penetration enhancers in human skin. J. Control. Release, 6, 85-97 (1987).

26) Kirschner N, Houdek P, Fromm M, Moll I, Brandner JM. Tight junctions form a barrier in human epidermis. Eur. J. Cell Biol., 89, 839-842 (2010).

27) Andrews SN, Jeong E, Prausnitz MR. Transdermal delivery of molecules is limited by full epidermis, not just stratum corneum. Pharm. Res., 30, 1099-1109 (2013). 